Mathematical Models and Methods in Applied Sciences

Vol. 20, No. 12 (2010) 2371-2374

(c) World Scientific Publishing Company

DOI: $10.1142 / \mathrm{S} 0218202510005306$

\title{
AUTHOR INDEX Volume 20
}

Amar, M., De Cicco, V. \& Fusco, N., Lower semicontinuity results for free discontinuity energies

Anaya, V., Bendahmane, M. \& Sepúlveda, M., A numerical analysis of a reaction-diffusion system modeling the dynamics of growth tumors

Arne, W., Marheineke, N., Meister, A. \& Wegener, R., Numerical analysis of Cosserat rod and string models for viscous jets in rotational spinning processes

Auricchio, F., Da Veiga, L. B., Hughes, T. J. R., Reali, A. \& Sangalli, G., Isogeometric collocation methods

Auricchio, F., Bonetti, E. \& Marigonda, A., A metricapproach to plasticity via Hamilton-Jacobi equation

Baffico, L., Grandmont, C. \& Maury, B., Multiscale modeling of the respiratory tract

Barrett, J. W. \& Prigozhin, L., A quasi-variational inequality problem in superconductivity

Baxevanis, Th., Katsaounis, Th. \& Tzavaras, A. E., Adaptive finite element computations of shear band formation

Bellomo, N., Berestycki, H., Brezzi, F. \& Nadal, J.-P., Mathematics and complexity in life and human sciences

Bellomo, N., Bellouquid, A., Nieto, J. \& Soler, J., Multiscale biological tissue models and flux-limited chemotaxis for multicellular growing systems

Bellouquid, A., see Bellomo

Bellouquid, A., On the asymptotic analysis of the BGK model toward the incompressible linear Navier-Stokes equation

Bendahmane, M., see Anaya Berestycki, H., see Bellomo

20 (2010) 707

20 (2010) 731

$20(2010) 1941$

20 (2010) 2075

20 (2010) 1617

20 (2010) 59

20 (2010) 679

20 (2010) 423

20S (2010) 1391

20 (2010) 1179

20 (2010) 1179

20 (2010) 1299
Bertozzi, A., see Rodriguez

Bonetti, E., see Auricchio

Bösing, P. R., Madureira, A. L. \& Mozolevski, I., A new interior penalty discontinuous Galerkin method for the Reissner- Mindlin model

Boutin, B., Chalons, C. \& Raviart, P.-A., Existence result for the coupling problem of two scalar conservation laws with Riemann initial data

Brantingham, P. J., see Jones

Brezzi, F., see Bellomo

Briane, M., Homogenization of the magneto-resistance in dimension two

Burman, E. \& Stamm, B., Bubble stabilized discontinuous Galerkin method for Stokes' problem

Buttà, P. \& Negrini, P., Directed current in quasi-adiabatically ac-driven nonlinear systems

Caglioti, E., see Pompei

Cardone, G., Panasenko, G. P. \& Sirakov, Y., Asymptotic analysis and numerical modeling of mass transport in tubular structures

Carrillo, J. A., Klar, A., Martin, S. \& Tiwari, S., Self-propelled interacting particle systems with roosting force

Casado-Díaz, J., Luna-Laynez, M. \& Suárez-Grau, F. J., Asymptotic behavior of a viscous fluid with slip boundary conditions on a slightly rough wall

Cavallaro, G. \& Marchioro, C., On the approach to equilibrium for a pendulum immersed in a Stokes fluid
20 (2010) 731

20S (2010) 1391

20S (2010) 1425 20 (2010) 1617

20 (2010) 1343

20 (2010) 1859

20S (2010) 1397

20S (2010) 1391

20 (2010) 1161

20 (2010) 297

20 (2010) 223

20S (2010) 1511

20 (2010) 397

20S (2010) 1533

20 (2010) 121

20 (2010) 1999 
Cavagna, A., Cimarelli, A., Giardina, I., Parisi, G., Santagati, R., Stefanini, F. \& Tavarone, R., From empirical data to interindividual interactions: Unveiling the rules of collective animal behavior

Chalons, C., Coquel, F. Godlewski, E., Raviart, P.-A \& Seguin, N., Godunov-type schemes for hyperbolic systems with parameter-dependent source. The case of Euler system with friction

Chalons, C., see Boutin

Charalambakis, N. \& Murat, F., Stability by homogenization of thermoviscoplastic problems

Chayes, L. R., see Jones

Choquet, C., Parabolic and degenerate parabolic models for pressure-driven transport problems

Cimarelli, A., see Cavagna

Colli, P., Gilardi, G., Podio-Guidugli, P. \& Sprekels, J., Existence and uniqueness of a global-in-time solution to a phase segregation problem of the Allen-Cahn type

Coquel, F., see Chalons

Coti Zelati, M., Giorgi, C.\& Pata, V. Steady states of the hinged extensible beam with external load

Da Veiga, L. B., see Auricchio

Dauge, M. \& Faou, E., Koiter estimate revisited

De Cicco, V., see Amar

Degond, P. \& Yang, T., Diffusion in a continuum model of selfpropelled particles with alignment interaction

Douglas, Jr., J., Spagnuolo, A. M. \& Yi, S.-Y., The convergence of a multidimensional, locally conservative Eulerian-Lagrangian finite element method for a semilinear parabolic equation

Doumic Jauffret, M. \& Gabriel, P., Eigenelements of a general aggregation-fragmentation model

Droniou, J., Eymard, R., Gallouët, T. \& Herbin, R., A unified approach to mimetic finite difference, hybrid finite volume and mixed finite volume methods

Durán, R. G. \& López García, F., Solutions of the divergence and analysis of the Stokes equations in planar Hölder- $\alpha$ domains

Eftang, J. L. \& Rønquist, E. M., Evaluation of flux integral outputs for the reduced basis method
Engström, C., On the spectrum of a holomorphic operator-valued function with applications to

20S (2010) 1491

20 (2010) 2109

20 (2010) 1859

20 (2010) 1591

20S (2010) 1397

20 (2010) 543

20S (2010) 1491

20 (2010) 519

20 (2010) 2109

20 (2010) 43

20 (2010) 2075

$20(2010) 1$

20 (2010) 707

20S (2010) 1459

$20(2010) 315$

20 (2010) 757

20 (2010) 265

$20(2010) 95$

20 (2010) 351 viscous shallow water equations absorptive photonic crystals

Eymard, R., see Droniou

Faou, E., see Dauge

Feireisl, E., Petzeltová, H., Rocca, E. \& Schimperna, G., Analysis of a phase-field model for twophase compressible fluids

Fellner, K. \& Raoul, G., Stable stationary states of non-local interaction equations

Formaggia, L., Minisini, S. \& Zunino, P., Modeling polymeric controlled drug release and transport phenomena in the arterial tissue

20 (2010) 1319

20 (2010) 265

20 (2010) 1

$20(2010) 1129$

$20(2010) 2267$

20 (2010) 1759

Frank, M., Herty, M. \& Sandjo, N. A., Optimal radiotherapy treatment planning governed by kinetic equations

Franzone, P. C., see Scacchi

Fusco, N., see Amar

Gabetta, E. \& Regazzini, E., About the gene families size distribution in a recent model of genome evolution

Gabriel, P., see Doumic Jauffret

Gallouët, T., see Droniou

Giardina, I., see Cavagna

Gilardi, G., see Colli

Giorgi, C., see Coti Zelati

Giovangigli, V. \& Tran, B., Mathematical analysis of a Saint-Venant model with variable temperature

Gittelson, C. J., Stochastic Galerkin discretization of the log-normal isotropic diffusion problem

Gobbi, R., Palpacelli, S. \& Spigler, R., Numerical treatment of a nonlinear nonlocal transport equation modeling crystal precipitation

Godlewski, E., see Chalons

Grandmont, C., see Baffico

Grasselli, M. \& Pierre, M., A splitting method for the CahnHilliard equation with inertial term

Halpern, L. \& Szeftel, J., Optimized and quasi-optimal Schwarz waveform relaxation for the one-dimensional Schrödinger equation

Haspot, B., Cauchy problem for with a term of capillarity

Hawkins, A., see Oden

Herbin, R., see Droniou
20 (2010) 661

20 (2010) 1089

20 (2010) 707

20 (2010) 1005

20 (2010) 757

20 (2010) 265

20S (2010) 1491

20 (2010) 519

20 (2010) 43

20 (2010) 1251

20 (2010) 237

20 (2010) 349

20 (2010) 2109

20 (2010) 59

$20(2010) 1363$

20 (2010) 2167

20 (2010) 1049

20 (2010) 477

20 (2010) 265 
Herty, M., see Frank

Hong, K.-I., see Neff

Hua, J. \& Yang, T., A note on the new Glimm functional for general systems of hyperbolic conservation laws

Huang, L., see Qin

Hughes, T. J. R., see Auricchio

Jeong, J., see Neff

Jones, P. A., Brantingham, P. J. \& Chayes, L. R., Statistical models of criminal behavior: The effects of law enforcement actions

Ju, Q. \& Wang, S., Quasi-neutral limit of the multidimensional drift-diffusion models for semiconductors

Katsaounis, Th., see Baxevanis

Kawashima, S., Nakamura, T., Nishibata, S. \& Zhu, P., Stationary waves to viscous heat-conductive gases in half-space: Existence, stability and convergence rate

Klar, A., see Carrillo

Knees, D., On global spatial regularity and convergence rates for time-dependent elasto-plasticity

Krzyżanowski, P., Laurençot, Ph. \& Wrzosek, D., Mathematical models of receptor-mediated transport of morphogens

Lachapelle, A., Salomon, J. \& Turinici, G., Computation of mean field equilibria in economics

Langwallner, B., Ortner, C. \& Süli, E., Existence and convergence results for the Galerkin approximation of an electronic density functional

Larsen, C. J., Ortner, C. \& Süli, E., Existence of solutions to a regularized model of dynamic fracture

Lattanzio, C.\& Piccoli, B., Coupling of microscopic and macroscopic traffic models at boundaries

Laurençot, Ph., see Krzyżanowski

Lescarret, V., Intermediate model for spatial evolution in nonlinear optics

Li, T. \& Wang, Z.-A., Nonlinear stability of large amplitude viscous shock waves of a generalized hyperbolic-parabolic system arising in chemotaxis

Lițcanu, G. \& Morales-Rodrigo, C., Asymptotic behavior of global solutions to a model of cell invasion

López García, F., see Durán
20 (2010) 661

20 (2010) 1553

20 (2010) 815

20 (2010) 589

20 (2010) 2075

20 (2010) 1553

20S (2010) 1397

20 (2010) 1649

20 (2010) 423

20 (2010) 2201

20S (2010) 1533

20 (2010) 1823

20 (2010) 2021

20 (2010) 567

20 (2010) 2237

20 (2010) 1021

20 (2010) 2349

20 (2010) 2021

20 (2010) 1209

20 (2010) 1967

20 (2010) 1721

20 (2010) 95
Loreto, V., see Pompei

Lorz, A., Coupled chemotaxis fluid model

Lube, G. \& Tews, B., Optimal control of singularly perturbed advection-diffusion-reaction problems

Luna-Laynez, M., see CasadoDíaz

Madureira, A. L., see Bösing

Marchioro, C., see Cavallaro

Marciniak-Czochra, A. \& Ptashnyk, M., Boundedness of solutions of a haptotaxis model

Marheineke, N., see Arne

Marigonda, A., see Auricchio

Martin, S., see Carrillo

Mathiaud, J., Local smooth solutions of a thin spray model with collisions

Maury, B., see Baffico

Maury, B., Roudneff-Chupin, A. \& Santambrogio, F., A macroscopic crowd motion model of gradient flow type

Mauser, N. J. \& Trabelsi, S., $L^{2}$ analysis of the multi-configuration time-dependent HartreeFock equations

Meirmanov, A., Double porosity models for liquid filtration in incompressible poroelastic media

Meister, A., see Arne

Minisini, S., see Formaggia

Morales-Rodrigo, C., see Lițcanu

Mozolevski, I., see Bösing

Mucha, P. B. \& Pokorný, M., Weak solutions to equations of steady compressible heat conducting fluids

Munnier, A. \& Pinçon, B., Locomotion of articulated bodies in an ideal fluid: 2D model with buoyancy, circulation and collisions

Murat, F., see Charalambakis

Nadal, J.-P., see Bellomo

Nakamura, T., see Kawashima

Neff, P., Hong, K.-I. \& Jeong, J., The Reissner-Mindlin plate is the $\Gamma$-limit of Cosserat elasticity

Negrini, P., see Buttà

Nieto, J., see Bellomo

Nishibata, S., see Kawashima

Nishibata, S., Shigeta, N. \& Suzuki, M., Asymptotic behaviors and classical limits of solutions to a quantum drift-diffusion model for semiconductors
20S (2010) 1511

20 (2010) 987

20 (2010) 375

20 (2010) 121

20 (2010) 1343

20 (2010) 1999

20 (2010) 449

20 (2010) 1941

20 (2010) 1617

20S (2010) 1533

20 (2010) 191

20 (2010) 59

20 (2010) 1787

20 (2010) 2053

20 (2010) 635

20 (2010) 1941

20 (2010) 1759

20 (2010) 1721

20 (2010) 1343

20 (2010) 785

20 (2010) 1899

20 (2010) 1591

20S (2010) 1391

20 (2010) 2201

20 (2010) 1553

20 (2010) 223

20 (2010) 1179

20 (2010) 2201

20 (2010) 909 
Oden, J. T., Hawkins, A. \& Prudhomme, S., General diffuse-interface theories and an approach to predictive tumor growth modeling

Ortner, C., see Langwallner

Ortner, C., see Larsen

Palpacelli, S., see Gobbi

Panasenko, G. P., see Cardone

Parisi, G., see Cavagna

Pata, V., see Coti Zelati

Pavarino, L. F., see Scacchi

Peletier, M. A. \& Veneroni, M., Stripe patterns in a model for block copolymers

Petzeltová, H., see Feireisl

Piccoli, B., see Lattanzio

Pierre, M., see Grasselli

Pinçon, B., see Munnier

Podio-Guidugli, P., see Colli

Pokorný, M., see Mucha

Pompei, S., Caglioti, E., Loreto, V. \& Tria, F., Distance-based phylogenetic algorithms: New insights and applications

Prigozhin, L., see Barrett

Prudhomme, S., see Oden

Ptashnyk, M., see MarciniakCzochra

Qin, Y. \& Huang, L., Regularity and exponential stability of the $p$ th Newtonian fluid in one space dimension

Raoul, G., see Fellner

Raviart, P.-A., see Boutin

Raviart, P.-A., see Chalons

Reali, A., see Auricchio

Rebholz, L. G. \& Sussman, M. M., On the high accuracy NS-alphadeconvolution turbulence model

Regazzini, E., see Gabetta

Rocca, E., see Feireisl

Rodriguez, N. \& Bertozzi, A. Local existence and uniqueness of solutions to a PDE model for criminal behavior

Rognes, M. E. \& Winther, R. Mixed finite element methods for linear viscoelasticity using weak symmetry

Rønquist, E. M., see Eftang

Rosier, L. \& Zhang, B.-Y., Control and stabilization of the nonlinear Schrödinger equation on rectangles

Roudneff-Chupin, A., see Maury

Salomon, J., see Lachapelle

Sandjo, N. A., see Frank

Sangalli, G., see Auricchio
20 (2010) 477

20 (2010) 2237

20 (2010) 1021

20 (2010) 349

20 (2010) 397

20S (2010) 1491

20 (2010) 43

20 (2010) 1089

20 (2010) 843

20 (2010) 1129

20 (2010) 2349

20 (2010) 1363

20 (2010) 1899

20 (2010) 519

20 (2010) 785

20S (2010) 1511

20 (2010) 679

20 (2010) 477

20 (2010) 449

20 (2010) 589

20 (2010) 2267

20 (2010) 1859

20 (2010) 2109

20 (2010) 2075

20 (2010) 611

20 (2010) 1005

20 (2010) 1129

20S (2010) 1425

20 (2010) 955

20 (2010) 351

20 (2010) 2293

20 (2010) 1787

20 (2010) 567

20 (2010) 661

20 (2010) 2075
Santagati, R., see Cavagna

Santambrogio, F., see Maury

Scacchi, S., Franzone, P. C., Pavarino, L. F. \& Taccardi, B., Computing cardiac recovery maps from electrograms and monophasic action potentials under heterogeneous and ischemic conditions

Schimperna, G., see Feireisl

Schoof, C., Coulomb friction and other sliding laws in a higherorder glacier flow model

Seguin, N., see Chalons

Sepúlveda, M., see Anaya

Shigeta, N., see Nishibata

Sirakov, Y., see Cardone

Süli, E., see Langwallner

Süli, E., see Larsen

Soler, J., see Bellomo

Spagnuolo, A. M., see Douglas, Jr.

Spigler, R., see Gobbi

Sprekels, J., see Colli

Stamm, B., see Burman

Stefanini, F., see Cavagna

Stuart, C. A. \& Zhou, H.-S., Existence of guided cylindrical TM-modes in an inhomogeneous self-focusing dielectric

Suárez-Grau, F. J., see CasadoDíaz

Sussman, M. M., see Rebholz

Suzuki, M., see Nishibata

Szeftel, J., see Halpern

Taccardi, B., see Scacchi

Tavarone, R., see Cavagna

Tews, B., see Lube

Tiwari, S., see Carrillo

Trabelsi, S., see Mauser

Tran, B., see Giovangigli

Tria, F., see Pompei

Turinici, G., see Lachapelle

Tzavaras, A. E., see Baxevanis

Veneroni, M., see Peletier

Wang, S., see Ju

Wang, Z.-A., see Li

Wegener, R., see Arne

Winther, R., see Rognes

Wrzosek, D., see Krzyżanowski

$\mathrm{Xu}$, J., Energy-transport limit of the hydrodynamic model for semiconductors

Yang, T., see Degond

Yang, T., see Hua

Yi, S.-Y., see Douglas, Jr.

Zhang, B.-Y., see Rosier

Zhou, H.-S., see Stuart

Zhu, P., see Kawashima

Zunino, P., see Formaggia
20S (2010) 1491

20 (2010) 1787

20 (2010) 1089

20 (2010) 1129

20 (2010) 157

20 (2010) 2109

20 (2010) 731

20 (2010) 909

20 (2010) 397

20 (2010) 2237

20 (2010) 1021

20 (2010) 1179

20 (2010) 315

20 (2010) 349

20 (2010) 519

20 (2010) 297

20S (2010) 1491

20 (2010) 1681

20 (2010) 121

20 (2010) 611

20 (2010) 909

20 (2010) 2167

20 (2010) 1089

20S (2010) 1491

20 (2010) 375

20S (2010) 1533

20 (2010) 2053

20 (2010) 1251

20S (2010) 1511

20 (2010) 567

20 (2010) 423

20 (2010) 843

20 (2010) 1649

20 (2010) 1967

20 (2010) 1941

20 (2010) 955

20 (2010) 2021

20 (2010) 937

20S (2010) 1459

20 (2010) 815

20 (2010) 315

20 (2010) 2293

20 (2010) 1681

20 (2010) 2201

20 (2010) 1759 